\title{
Stem Cell Signaling in Immunity and Development
}

\author{
H. Lee, O.-K. Chah, J. Plotnikov, and J. Sheen \\ Department of Molecular Biology, and Center for Computational and Integrative Biology, \\ Massachusetts General Hospital, Boston, Massachusetts 02114; Department of Genetics, Harvard \\ Medical School, Boston, Massachusetts 02114 \\ Correspondence: sheen@molbio.mgh.harvard.edu
}

\begin{abstract}
Stem cells in the shoot apical meristem (SAM) of plants are the self-renewable reservoir for leaf, stem, and flower organogenesis. Stem-cell fate and population size are subject to regulation by complex intrinsic signals and environmental cues to ensure balanced plant development, survival, and longevity. Peptides secreted from the shoot stem cells have pivotal roles in controlling cell identity, proliferation, and differentiation through multiple receptor kinase complexes. The best-characterized in vivo and in vitro peptide ligands are the 12-amino acid (aa) and the arabinosylated 13-aa CLAVATA3 peptides (CLV3p) that are perceived by multiple receptors with partially overlapping and distinct expression patterns and functions in the SAM. The primary molecular and cellular signaling mechanisms after the occurrence of ligand-receptor interaction remain elusive. Integrated analyses provide novel evidence for differential peptide-receptor signaling in the dynamic regulation of stem-cell homeostasis and fitness. Surprisingly, the 12-aa CLV3p can trigger immune signaling and limit pathogen invasion via the flagellin receptor kinase FLS2, suggesting a previously unrecognized molecular mechanism underlying enhanced immunity in the SAM area. Because pattern recognition receptor signaling in immune responses also profoundly intercepts plant development, peptide-receptor kinase signaling in immunity and development may share a common evolutionary origin.
\end{abstract}

Plant growth and development are sustained by shoot and root meristems that are fully established after germination and are controlled by integrated signaling networks based on a developmental genetic blueprint subjected to dynamic and profound regulation by nutrients, hormones, and environmental signals. Pluripotent stem cells are maintained in specific niches in the meristems and supply new cells for potentially infinite and plastic growth, a unique characteristic of plant development. Stem-cell fate and population size are subject to complex regulation by intrinsic signals and environmental cues to control the balance between proliferation and differentiation. Molecular genetic and biochemical advances during past decades have revealed that peptides secreted and processed from and around stem cells have pivotal roles in controlling cell identity and developmental processes through multiple receptor kinase complexes in the SAM (Fig. 1) (Kondo et al. 2006; DeYoung and Clark 2008; Jun et al. 2008; De Smet et al. 2009; Guo et al. 2010; Kinoshita et al. 2010; Gish and Clark 2011; Matsubayashi 2011; Aichinger et al. 2012; Perales and Reddy 2012; Song et al. 2012). However, little is known about the dynamic biochemical, molecular, and cellular events and regulatory components constituting the primary signaling processes after peptide ligand perception by receptor kinases in the SAM (Betsuyaku et al. 2011; Perales and Reddy 2012). It has also been enigmatic how the stem cells in the meristems sustain longevity and are protected from damages by environmental stresses and pathogen attacks (Hollings 1965).

Integrative molecular, cellular, biochemical, genetic, and genomic approaches have been indispensible in un- raveling the primary peptide-receptor signaling networks in plant innate immune signaling, which orchestrate diverse cellular functions and processes controlling both defense and development (Asai et al. 2002; Boller and Felix 2009; Boudsocq et al. 2010; Tena et al. 2011). Because peptide-receptor signaling pathways in various biological contexts may use evolutionarily conserved components and analogous mechanisms (Fig. 2), advances in the development of technical tools and cellular assays in conceptual and mechanistic understanding of immune responses could prove to be valuable in helping to uncover new insight in developmental processes controlled by stem cells restricted to the meristems.

\section{CLV3P-MEDIATED SAM IMMUNITY}

To study novel peptide-receptor interactions and the primary events in plant peptide-receptor signaling, we developed a cell-based plant expression system to analyze peptide-mediated receptor-like kinase (RLK) signaling (Asai et al. 2002; Lee et al. 2011) and to screen for new signaling peptides and corresponding receptors. We observed that the endogenously modified 12-amino-acid (aa) CLV3p triggered similar responses to flg22 (the conserved 22-aa peptide of bacterial flagellin) in mesophyll protoplasts (Asai et al. 2002; Shan et al. 2008; Lee et al. 2011). This finding was unexpected because CLV3p is normally expressed, secreted, and processed by stem cells to control SAM maintenance via CLV1, CLV2, and other receptors (Kondo et al. 2006; Jun et al. 2008; Gish and Clark 2011; Matsubayashi 2011; Aichinger et al. 2012). Flg22 and CLV3p, but not $\triangle$ CLV3p lacking the last 12th 
A
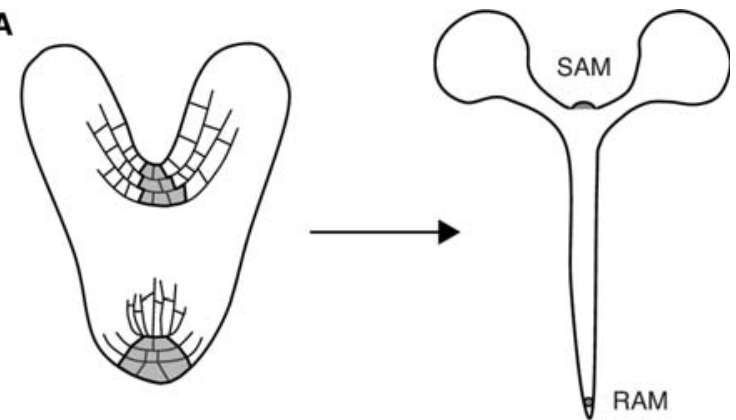

B

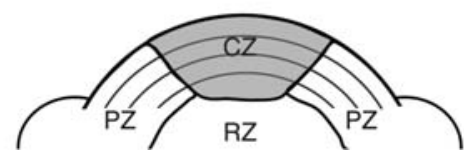

Figure 1. Schemes of shoot apical meristem (SAM) in plant development. (A) Postembryonic development in plants. Meristems (gray) that contain stem-cell population support de novo growth and development after embryogenesis and germination. Plant primary meristems are located at the tip of shoot and root apex, SAM, and RAM (root apical meristem), respectively. The location and population size of meristems are maintained throughout the lifespan from embryos (right) to seedlings (right) and adult plants. $(B)$ Structure of a SAM. The SAM is functionally separated into the central zone (CZ), peripheral zone (PZ), and rib zone (RZ). Stem cells are located in the $\mathrm{CZ}$ (gray) and replenish new PZ and RZ progenitor cells to generate lateral organs and stem.

His residue, activated similar mitogen-activated protein kinase (MAPK) activities and multiple primary immune response marker genes (Asai et al. 2002; He et al. 2006; Shan et al. 2008; Boller and Felix 2009; Boudsocq et al. 2010). Highly purified CLV3p synthesized by different

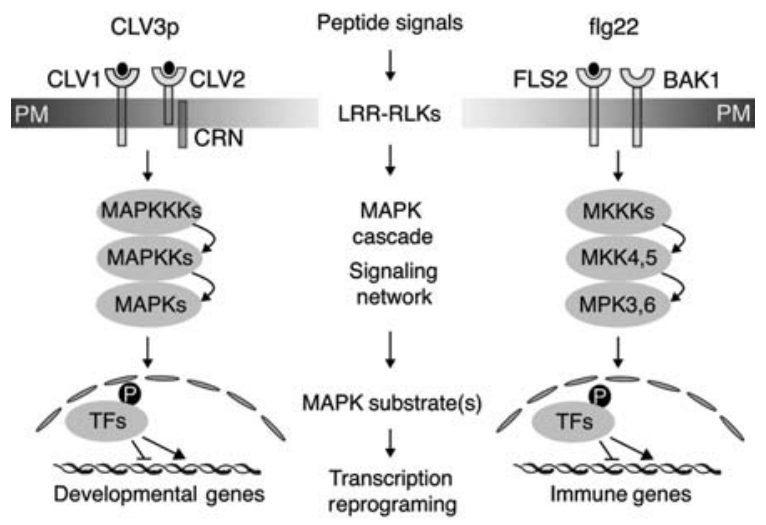

Figure 2. Proposed conservation of peptide-mediated receptorlike kinase signaling in immunity and development. CLV3 peptide (CLV3p) secreted from stem cells and flg22 peptide of bacterial flagellin are recognized by CLV1-CLV2/CRN receptor complex and FLS2, respectively. After flg22 perception, FLS2 interacts with RLK BAK1 and triggers downstream mitogen-activated protein kinase (MAPK) cascade to phosphorylate transcription factors (TFs) that can activate or repress immune response gene transcription. CLV1 and FLS2 encode highly conserved LRR-RLKs. MAPK cascade could be used in CLV3p-mediated CLV1-CLV2/CRN signaling to activate or repress developmental genes. (LRR-RLK) Leucine-rich-repeat receptor-like kinase. sources displayed the same activities, ruling out the possibility of contamination. Neither the dominant clv1-1 mutant nor the clv2-1 mutant affected CLV3p-triggered MAPK activation. Surprisingly, two independent Ler and Col-0 fls 2 mutant alleles, but not efr-1 (the bacterial elongation factor EF-Tu receptor EFR) mutant, failed to support the activation of MAPKs by both flg22 and CLV3p. Complementation with the FLS2 gene but not CLV1 in the fls 2 mutant confirmed that FLS2 could recognize both flg22 and CLV3p to mediate MAPK signaling (Lee et al. 2011).

Because CLV3p is specifically expressed and secreted from stem cells of the SAM (Kondo et al. 2006; Jun et al. 2008; Gish and Clark 2011; Matsubayashi 2011; Aichinger et al. 2012), it is critical to examine CLV3pFLS2 signaling in the SAM to evaluate its physiological relevance. Using quantitative reverse transcription-polymerase chain reaction (qRT-PCR) analysis with isolated SAM tissues, CLV3p clearly triggered two parallel signaling pathways in the SAM. The activation of important immune response marker genes by CLV3p in wild-type (WT), clv1-1, and $c l v 2-1$ but not $f l s 2-24$ validated the action of innate immune signaling via FLS2 in the Arabidopsis SAM. These flg22 and CLV3p inducible genes have important roles in bacterial and fungal resistance (Asai et al. 2002; Boller and Felix 2009; Boudsocq et al. 2010). Consistently, some of these immune response marker genes showed reduced endogenous expression in the SAM of $\operatorname{clv} 3-2$ but were constitutively expressed at higher levels in the SAM but not other tissues of the WT. Their endogenous expression levels were low in both the SAM and other tissues in $c l v 3-2$ and $f l s 2-$ 24. Complementation of $\mathrm{clv} 3-2$ for immune marker gene expression in the SAM could be achieved within the nanomolar range of exogenous CLV3p, reflecting the physiological relevance of the estimated dissociation constant $\left(K_{\mathrm{d}}\right)(34.7 \mathrm{nM})$ for CLV3p and FLS2 interaction and CLV3p-FLS2 signaling in the SAM (Lee et al. 2011).

Using laser-scanning confocal microscopy, we could confidently visualize Pst DC3000-GFP (green fluorescent protein) infection inside plant tissues by serial optimal sections by moving the focus point away from the plant surface. Most strikingly, we had never detected the presence of a single live Pst DC3000-GFP bacterium in the SAM of WT seedlings for up to $4 \mathrm{~d}$ after infection in numerous experiments. Because Pst DC3000-GFP infection occurred in both WT and clv3-2 cotyledons, the WT SAM appeared to exhibit differential immunity. Significantly, the SAM was no longer protected from Pst DC3000-GFP infection in fls2-24 and clv3-2 mutants, supporting the important role of the endogenous CLV3p in SAM protection by FLS2-mediated innate immune signaling pathway (Lee et al. 2011, 2012). To further demonstrate and quantify the proliferation and growth of $P s t$ DC3000-GFP in the SAM of fls2-24 and clv3-2 mutants, we performed quantitative PCR (qPCR) analysis of the GFP DNA from the bacteria, indicating the loss of distinct SAM immunity. Importantly, Pst DC3000-GFP was found to be completely excluded from the similarly enlarged clv1-1 and clv2-1 SAM, in which CLV3p-FLS2 


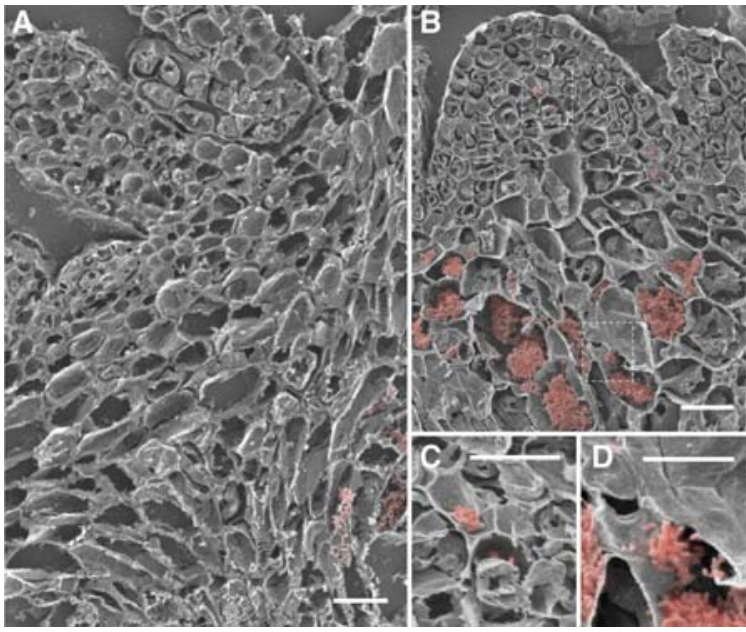

Figure 3. Analysis of Pst DC3000 infection in SAM regions by scanning electron microscopy (SEM) with tissue cross sections. (A) Immune protection of the Ler SAM area. Show is a cross section through the SAM area. Multiple cell layers below the SAM did not reveal any Pst DC3000 bacteria (4 dpi [days postinfection]), whereas invaded bacteria (pink) were observed many cell layers away from the SAM. (B) Compromised immune protection in the $c l v 3 \mathrm{SAM}$ area. Pst DC3000 colonized in the SAM and cells below the SAM ( 4 dpi). $(A, B)$ Scale bars, $20 \mu \mathrm{m}$. $(C, D)$ Magnified images of selected upper and lower regions. ( $B$, white lined boxes) Bacterial colonization. $(C, D)$ Scale bars, $10 \mu \mathrm{m}$.

signaling remained active. On the basis of qPCR analysis of GFP DNA derived only from Pst DC3000-GFP, nonspecific bacterial attachment background could be estimated from the SAM tissue samples $1 \mathrm{~h}$ after bacteriaseedling cocultivation. Consistent with confocal microscopic observations, active bacterial growth and proliferation were exclusively detected only in the SAM of $\operatorname{clv} 3-2$ and fls2-24 mutants (Lee et al. 2011, 2012).

Because SAM inner tissues are not fully accessible to confocal microscopy-based imagining, we applied scanning electron microscopy (SEM) to analyze cross sections of SAM domes and surrounding cells of 4-dpi (days postinfection) seedlings (Plotnikova et al. 2000). SEM offered the possibility of revealing the spatial distribution of bacterial cells in the SAM and neighboring plant cells. We observed clear bacterial colonization of SAM cells or cells very close to the SAM in clv 3 but not Ler WT seedlings, in which we only observed invaded cells many cell layers away from the SAM (Fig. 3). These data suggest that CLV3p-based resistance of the WT SAM extends multiple cell layers below the SAM (Lee et al. 2012). However, in cotyledons and leaves distant from the SAM, the virulent bacterial pathogen Pst DC3000 successfully colonized in both WT and clv3 seedlings (Lee et al. 2011).

We have uncovered a surprising mechanism underlying stem-cell-triggered immunity for pathogenic bacteria protection through CLV3p-FLS2 signaling. It will be interesting to examine SAM protection from a variety of other pathogens. We propose that CLV3p is recognized by two distinct types of receptors involved in mostly nonoverlapping functions in the SAM (Fig. 4). "Constitutive" immunity in the SAM resembles flg22 pretreatment as a type of vaccination (before pathogen inoculation), which is more effective in conferring protection against virulent pathogens such as $P$ st DC3000 possessing virulent effectors to cripple microbe-associated molecular patterns (MAMP) signaling (He et al. 2006; Shan et al. 2008; Boller and Felix 2009; Boudsocq et al. 2010; Lee et al. 2011).

\section{DIFFERENTIAL PEPTIDE-RECEPTOR SIGNALING}

In many studies using MAMPs, such as flg22 and elf18, innate immune responses and growth inhibition are always tightly linked (Boller and Felix 2009; Lee et al. 2011, 2012). Surprisingly, CLV3p did not inhibit shoot growth but caused stronger root growth arrest in Col-0 and Ler WT seedlings. Although seedling growth inhibition in both shoots and roots by flg22 was

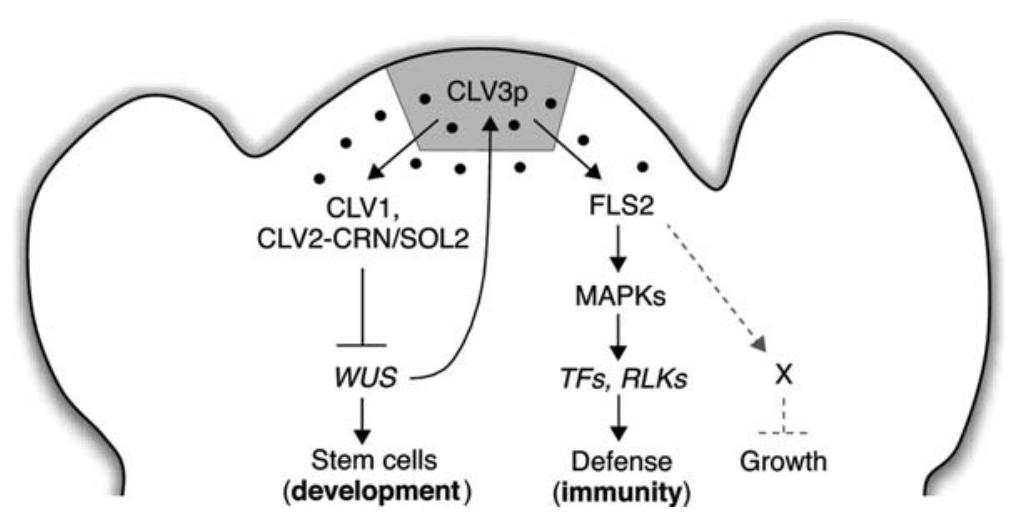

Figure 4. Model of CLV3p-FLS2 signaling in the SAM. CLV3p (black dots), expressed and secreted from stem cells (gray region), bind to the FLS2 receptor and activate MAPK cascade signaling and immune response marker genes. The novel CLV3p-FLS2 signaling pathway is nonoverlapping with CLV receptor complex-mediated stem-cell homeostasis. CLV3p did not stimulate the typical flg22-FLS2-mediated growth suppression in whole seedlings (gray dashed lines), which is triggered by unknown downstream components $(\mathrm{X})$. (TFs) Transcription factors, $(R L K s)$ receptor-like kinases. 
eliminated in two independent $f l s 2$ mutants, the stronger root growth arrest by CLV3p was retained. Although CLV3p activated, via FLS2, a spectrum of innate immune responses similar to those via flg22-FLS2 signaling in mesophyll cells, seedlings, and the SAM, CLV3p did not stimulate severe growth suppression in whole seedlings that was typical in flg22-FLS2-mediated responses. Similar to other synthetic CLAVATA3/embryo-surrounding region (CLE) peptides, CLV3p-triggered root growth arrest was partially abolished in the clv2-1 mutant (Miwa et al. 2008; Müller et al. 2008). Thus, CLV3pFLS2 signaling activated only immune responses but not the general growth inhibition (Fig. 4) (Lee et al. 2011, 2012).

Because positively charged amino-terminal Arg and carboxy-terminal His-His residues are essential for synthetic 12-aa MCLV3p activity (Kondo et al. 2006, 2008; Betsuyaku et al. 2011; Lee et al. 2011) and for transgenic CLV3 complementation in clv3 (Song et al. 2012), we hypothesized that CLV3p-FLS2 interactions may be $\mathrm{pH}$ dependent. Interestingly, CLV3p activity mediated through FLS2 was abolished at $\mathrm{pH} 7.6$ and was most active at $\mathrm{pH} 5-6$, reflecting the physiological apoplastic environment after protein secretion, whereas flg 22 activated similar responses from $\mathrm{pH} 5$ to 7.6 and peaked at $\mathrm{pH} 7$. Thus, to distinguish between flg22 signaling and CLV3p signaling, experiments using higher $\mathrm{pH}(7-7.6)$ can be recommended. Moreover, $1 \mu \mathrm{M}$ CLV3p did not block the activation of immune response genes when $1 \mathrm{nM}$ flg22 was spiked into the $\mathrm{pH} 7$ medium (when MCLV3p was inactive) (Lee et al. 2012). These new findings are consistent with the inability of CLV3p to suppress wholeseedling growth as flg22 and differential peptide binding competition with FLS2 and provide additional strong evidence that novel 12-aa MCLV3p activity is not the same as flg22 activity (Lee et al. 2011, 2012).

\section{COMMON ORIGINS OF RECEPTOR KINASES IN IMMUNITY AND DEVELOPMENT}

In both plants and animals, innate immunity provides the first line of inducible defense and is triggered through pattern recognition receptors (PRRs) in response to MAMPs (Ishii et al. 2008; Boller and Felix 2009). In plants, receptor kinases represent the main functions of known plasma membrane PRRs for MAMP recognition to distinguish nonself from self (Boller and Felix 2009). FLS2 is the first characterized plant leucine-rich-repeat (LRR) receptor kinase that perceives the bacterial flagellin and launches convergent downstream signaling and defense pathways for potentially broad-spectrum pathogen resistance (Gómez-Gómez and Boller 2000; Asai et al. 2002; Yamamizo et al. 2006; Boller and Felix 2009).

It is interesting to note that LRR receptor kinases involved in immunity are closely related to well-characterized LRR receptor kinases involved in development based on genome-wide comparative analysis of the RLK gene families in Arabidopsis and rice (Shiu and Bleecker 2003; Shiu et al. 2004; Lehti-Shiu et al. 2012). Because FLS2- and EFR-mediated signaling also strongly regulates plant growth and development (Boller and Felix 2009), it is evident that these PRRs have dual roles in immunity and development. Our findings with differential CLV3-FLS2 signaling further suggest the possibility of bifurcation in signaling processes downstream from the LRR receptor kinase, FLS2, in modulating immune and developmental responses (Fig. 4) (Lee et al. 2011). This is reminiscent of the dual functions of the Drosophila Toll receptor in dorsoventral patterning in embryo development and in adult fly innate immunity (Lemaitre et al. 1996).

The 12-aa CLV3p appears to be recognized by six distinct receptors (FLS2, CLV1, CLV2, RPK2, BAM1, and BAM2) based on genetic analyses and biochemical binding assays (Fig. 5) (Kondo et al. 2006; DeYoung and Clark 2008; Miwa et al. 2008; Müller et al. 2008; Ohyama et al. 2009; Guo et al. 2010; Kinoshita et al. 2010; Betsuyaku et al. 2011; Lee et al. 2011). Among these receptors, the LRR domains for ligand perception or the PK domains for cellular signaling are more closely related between CLV1 and FLS2 than between CLV1 and CLV2 or RPK2 (Fig. 6) (Shiu and Bleecker 2003; Shiu et al. 2004; Wang and Fiers 2010; Lehti-Shiu et al. 2012).

Moreover, both CLV1 involved in plant development and PEPR1/2 involved in plant defense responses belong to the same XI LRR-RLK subfamily, which is closest to the XII LRR-RLK subfamily including pattern recognition receptor kinases such as FLS2 and EFR (Table 1) (Shiu and Bleecker 2003; Shiu et al. 2004; Boller and Felix 2009; Krol et al. 2010; Qi et al. 2010; Wang and Fiers 2010; Yamaguchi et al. 2010; Lehti-Shiu et al. 2012). Interestingly, PEPR1 recognized six different peptides (Pep1-6) to activate immune response genes, whereas PEPR2 only responded to Pep1 -2 but had a predominant role in Pep-mediated growth repression (Krol et al. 2010; Yamaguchi et al. 2010). Another closely related LRRRLK in XIII LRR-RLK subfamily, ERECTA (ER), has been implicated in both development and defense (Llorente et al. 2005; Shapk et al. 2005). LRR-RLKs likely evolved from common origins to support complex signaling networks in development programs and immunity in multicellular plants.

\section{CONCLUSIONS}

Analogous to animal germ-line cells, which exhibit enhanced stress resistance and immunity (Curran et al. 2009), stem cells in the SAM are the source of plant germ-line cells and can remain protected and viable even when plant leaves are severely damaged by diverse stress challenges and bacterial and viral infections (Hollings 1965; Kovtun et al. 2000; Lee et al. 2011, 2012). CLV3p-FLS2 signaling might have evolved to provide constitutive immune protection in the SAM but avoid the penalty from growth suppression normally associated with potent MAMP-mediated immune signaling (Boller and Felix 2009). It will be interesting to elucidate the precise differential downstream signaling events via the same FLS2 receptor in response to different peptide 


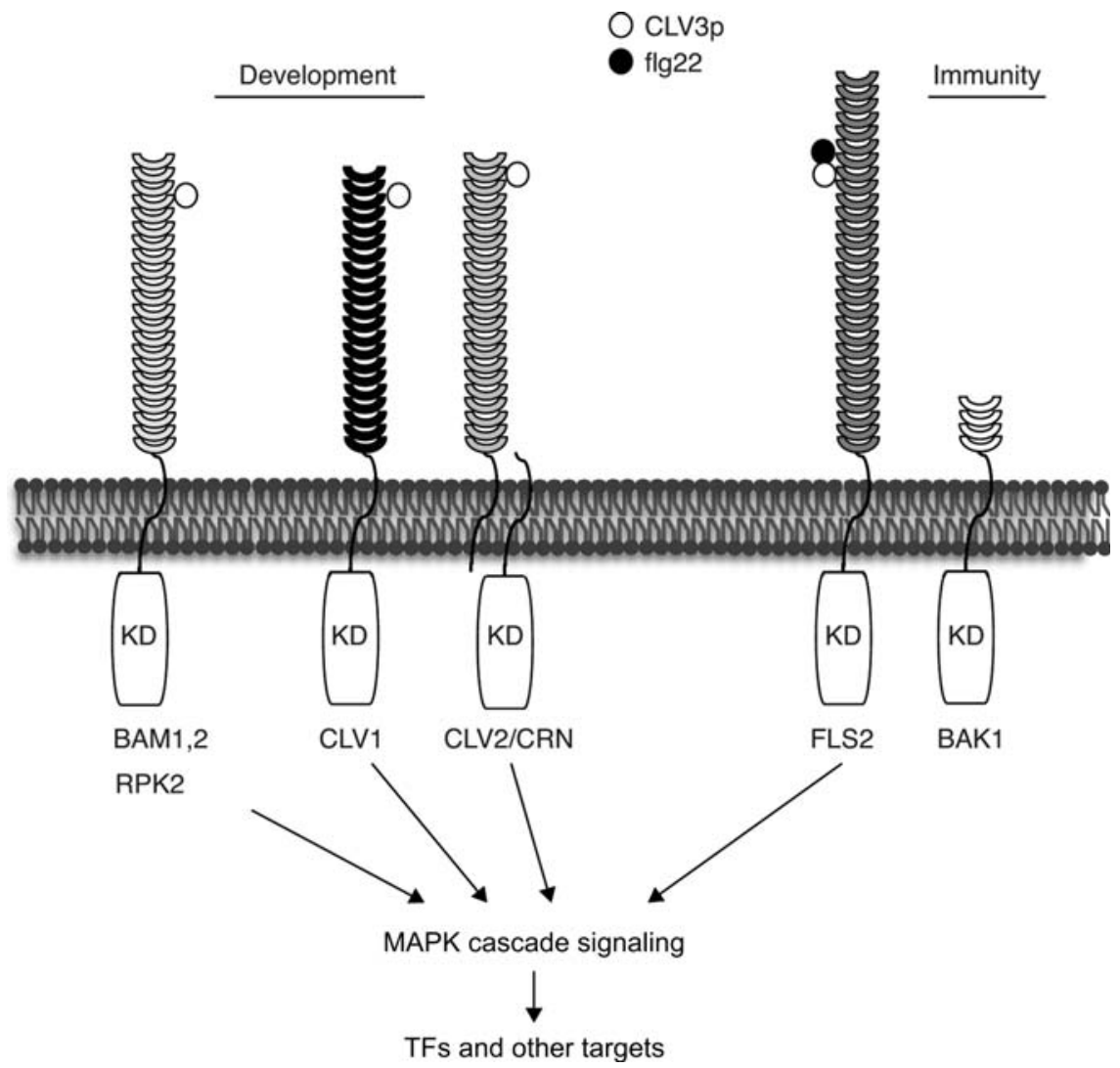

Figure 5. CLV3p receptors involved in development and immunity in the SAM. Five LRR-RLKs (CLV1, BAM1, BAM2, RPK2, and FLS2) and one LRR-RLP (CLV2) are implicated as CLV3 receptors via genetic and biochemical analyses. An LRR-RLK consists of three main domains: extracellular LRR, transmembrane, and intracellular kinase domain (KD). CRN (CORYNE) is an RLK that lacks an extracellular LRR domain but complexes with CLV2 lacking any intracellular KD. The binding sites of CLV3p and flg22 on the FLS2 receptor are partially overlapped. Receptor-mediated signaling typically activates MAPK cascades that regulate transcription factors (TFs) and other targets. (LRR-RLK) Leucine-rich-repeat receptor-like kinase, (LRR-RLP) LRR receptor-like protein.

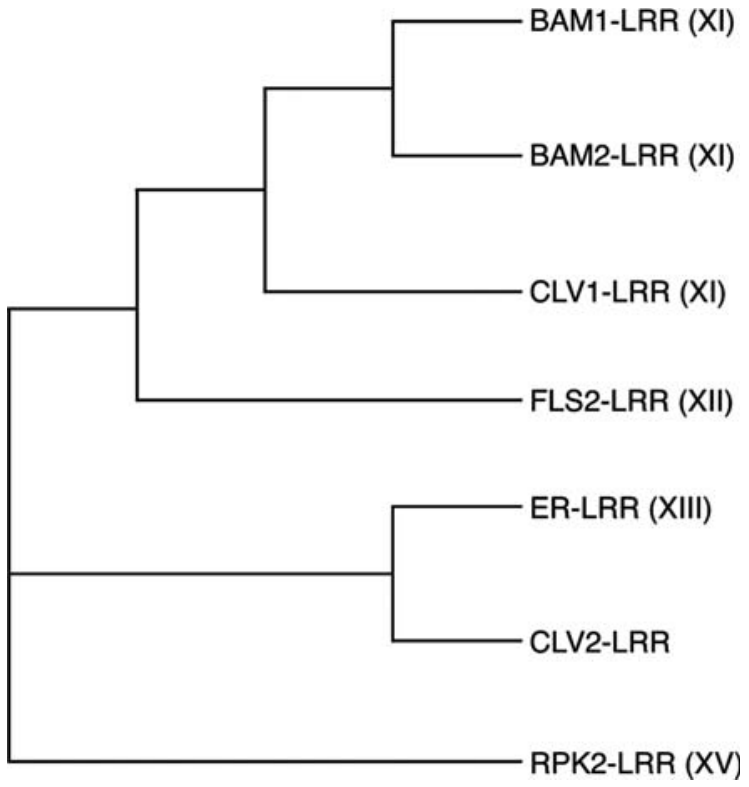

Figure 6. Analyses of sequence similarity among CLV3p receptors in the SAM. Protein sequence alignment of LRR domains was performed with the ClustalX program, and a tree showing genetic distance was generated by the NJplot program. The tree result was presented through the Dendroscope program (rectangular cladogram). The numbers in parentheses represent the subfamily in the LRR-RLK family. (LRR-RLK) Leucine-rich-repeat receptor-like kinase, LRR-RLP: LRR receptor-like protein. 
Table 1. Closely related peptide-receptor kinase signaling in plant development and immunity

\begin{tabular}{lllll}
\hline & Subfamily & Receptor(s) & Peptide ligand(s) & $\begin{array}{c}\text { Length of } \\
\text { peptides } \\
\text { (amino acids) }\end{array}$ \\
\hline LRR-RLP & & CLV2 & CLV3p, 19 CLEps & $12-13$ \\
LRR-RLK & XI & PEPR1,2 & Pep1-6 & 23 \\
LRR-RLK & XI & CLV1, BAM1,2 & CLV3p & D \\
LRR-RLK & XII & FLS2 & flg15, flg22, CLV3p & $12-13$ \\
LRR-RLK & XII & EFR & elf18 & 18 \\
LRR-RLK & XII & ER, ERL1,2 & D I & D and I \\
LRR-RLK & XV & RPK2 & CLV3p & D and I \\
\hline
\end{tabular}

${ }^{\mathrm{a}}$ Peptide-mediated receptor kinase signaling functions in development (D) and/or immunity (I) in plants. (LRR-RLP) Leucine-rich-repeat receptorlike protein, (LRR-RLK) LRR receptor-like kinase.

Table data from Rehmany et al. (2005), Fabro et al. (2011), and Cailland et al. (2012).

ligands. A general future challenge in the emerging plant peptide-receptor signaling field is to elucidate the precise differential downstream signaling events via the same or different receptors in response to the potentially large spectrum of peptide ligands in various posttranslational modification forms (Jun et al. 2008; Butenko et al. 2009; Ohyama et al. 2008; Matsubayashi 2011; Meng et al. 2012).

Lacking the genes for antibodies and immune cell receptors in humans (Goldsby et al. 2006) to respond to new signals from diverse invaders, plant RLKs, displaying high polymorphism and fast evolution (Clark et al. 2007; Lehti-Shiu et al. 2012), may provide an alternative means to recognize self or nonself in a beneficial manner through constant selections in evolution. It will also be important to explore the roles of other secreted plant peptides and known or orphan receptors in innate immunity and development (Jun et al. 2008; Ohyama et al. 2008; Butenko et al. 2009; De Smet et al. 2009; Matsubayashi 2011; Lehti-Shiu et al. 2012; Meng et al. 2012). Innovative and integrative approaches will facilitate matching, discriminating, and dissecting peptide-receptor signaling events and elucidating the physiological functions of plant RLKs and peptide signals.

\section{ACKNOWLEDGMENTS}

This work was supported by funding from the National Institute of Health (grants R01GM060493 and R01GM070567) and the National Science Foundation (grant IOS-0618292).

\section{REFERENCES}

Aichinger E, Kornet N, Friedrich T, Laux T. 2012. Plant stem cell niches. Annu Rev Plant Biol 63: 615-636.

Asai T, Tena G, Plonikova J, Willmann M, Chiu W-L, GomezGomez L, Boller T, Ausubel FM, Sheen J. 2002. MAP kinase signaling cascade in Arabidopsis innate immunity. Nature 415: $977-983$.

Betsuyaku S, Takahashi F, Kinoshita A, Miwa H, Shinozaki K, Fukuda H, Sawa S. 2011. Mitogen-activated protein kinase regulated by the CLAVATA receptors contributes to shoot apical meristem homeostasis. Plant Cell Physiol 52: 14-29.

Boller T, Felix G. 2009. A renaissance of elicitors: Perception of microbe-associated molecular patterns and danger signals by pattern-recognition receptors. Annu Rev Plant Biol 60: $379-406$.
Boudsocq M, Willmann MR, McCormack M, Lee H, Shan L, He P, Bush J, Cheng S-H, Sheen J. 2010. Differential innate immune signalling via $\mathrm{Ca}^{2+}$ sensor protein kinases. Nature 464: 418-422.

Butenko MA, Vie AK, Brembu T, Aalen RB, Bones AM. 2009. Plant peptides in signaling: Looking for new partners. TIPS 14: $255-263$.

Clark RM, Schweikert G, Toomajian C, Ossowski S, Zeller G, Shinn P, Warthmann N, Hu TT, Fu G, Hinds DA, et al. 2007. Common sequence polymorphisms shaping genetic diversity in Arabidopsis thaliana. Science 317: 338-342.

Curran SP, Wu X, Riedel CG, Ruvkun G. 2009. A soma-togermline transformation in long-lived Caenorhabditis elegans mutants. Nature 459: 1079-1084.

De Smet I, Voß U, Jürgens G, Beeckman T. 2009. Receptor-like kinases shape the plant. Nature Cell Biol 11: 1166-1173.

DeYoung BJ, Clark SE. 2008. BAM receptors regulate stem cell specification and organ development through complex interactions with CLAVATA signaling. Genetics 180: $895-$ 904.

Gish LA, Clark SE. 2011. The RLK/Pelle family of kinases. Plant J 66: 117-127.

Goldsby RA, Kindt TJ, Osborne BA. 2006. KUBY IMMUNOL$O G Y$, 6th ed. WH FREEMAN \& Co, New York.

Gómez-Gómez L, Boller T. 2000. FLS2: An LRR receptor-like kinase involved in the perception of the bacterial elicitor flagellin in Arabidopsis. Mol Cell 5: 1003-1011.

Guo Y, Han L, Hymes M, Denver R, Clark SE. 2010. CLAVATA2 forms a distinct CLE-binding receptor complex regulating Arabidopsis stem cell specification. Plant $J$ 63: 889-900.

He P, Shan L, Lin NC, Martin GB, Kemmerling B, Nurnberger T, Sheen J. 2006. Specific bacterial suppressors of MAMP signaling upstream of MAPKKK in Arabidopsis innate immunity. Cell 125: 563-575.

Hollings M. 1965. Disease control through virus-free stock. Annu Rev Phytopath 3: 367-396.

Ishii KJ, Koyama S, Nakagawa A, Coban C, Akira S. 2008. Host innate immune receptors and beyond: Making sense of microbial infections. Cell Host Microbe 3: 352-363.

Jun JH, Fiume E, Fletcher JC. 2008. The CLE family of plant polypeptide signaling molecules. Cell Mol Life Sci 65: $743-755$.

Kinoshita A, Betsuyaku S, Osakabe Y, Mizuno S, Nagawa S, Stahl Y, Simon R, Yamaguchi-Shinozaki K, Fukuda H, Sawa S. 2010. RPK2 is an essential receptor-like kinase that transmits the CLV3 signal in Arabidopsis. Development 137: $3911-3920$.

Kondo T, Sawa S, Kinoshita A, Mizuno S, Kakimoto T, Fukuda H, Sakagami Y. 2006. A plant peptide encoded by CLV3 identified by in situ MALDI-TPF MS analysis. Science 313: $845-848$.

Kondo T, Nakamura T, Yokomine K, Sakagami Y. 2008. Dual assay for MCLV3 activity reveals structure-activity relationship of CLE peptides. Biochem Biophys Res Commun 377: 312-316. 
Kovtun Y, Chiu W-L, Tena G, Sheen J. 2000. Functional analysis of oxidative stress-activated MAPK cascade in plants. Proc Natl Acad Sci 97: 2940-2945.

Krol E, Mentzel T, Chinchilla D, Boller T, Felix G, Kemmerling B, Postel S, Arents M, Jeworutzki E, Al-Rasheid KAS, et al. 2010. Perception of the Arabidopsis danger signal peptide 1 involves the pattern recognition receptor AtPEPR1 and its close homologue AtPEPR2. J Biol Chem 285: $13471-13479$

Lee H, Chah O-K, Sheen J. 2011. Stem-cell-triggered immunity through CLV3p-FLS2 signalling. Nature 473: 376-379.

Lee H, Khatri A, Plotnikov JM, Zhang X-C, Sheen J. 2012. Complexity in differential peptide-receptor signaling. Plant Cell 24: 3177-3185.

Lehti-Shiu MD, Zou C, Shiu SH. 2012. Origin, diversity, expansion history and functional evolution of the plant receptor-like Kinase/Pelle family. Signal Commun Plants 13: $1-22$.

Lemaitre B, Nicolas E, Michaut L, Reichhart J-M, Hoffmann JA. 1996. The dorsoventral regulatory gene cassettespätzle/ Toll/cactus controls the potent antifungal response in Drosophila adults. Cell 86: 973-983.

Llorente F, Alonso-Blanco C, Sánchez-Rodriguez C, Jorda L, Molina A. 2005. ERECTA receptor-like kinase and heterotrimeric G protein from Arabidopsis are required for resistance to the necrotrophic fungus Plectosphaerella cucumerina. Plant J 43: 165-180.

Matsubayashi Y. 2011. Small post-translationally modified peptide signals in Arabidopsis. The Arabidopsis Book, Vol. 9, Amer Soc Plant Biol.

Meng L, Buchanan BB, Feldman LJ, Luan S. 2012. CLE-like (CLEL) peptides control the pattern of root growth and lateral root development in Arabidopsis. Proc Natl Acad Sci 109: $1760-1765$.

Miwa H, Betsuyaku S, Iwamoto K, Kinoshita A, Fukuda H, Sawa S. 2008. The receptor-like kinase SOL2 mediates CLE signaling in Arabidopsis. Plant Cell Physiol 49: 1752-1757.

Müller R, Bleckmann A, Simon R. 2008. The receptor kinase CORYNE of Arabidopsis transmits the stem cell-limiting signal CLAVATA3 independently of CLAVATA1. Plant Cell 20: 934-946.

Ohyama K, Ogawa M, Matsubayashi Y. 2008. Identification of a biologically active, small, secreted peptide in Arabidopsis by in silico gene screening, followed by LC-MS-based structure analysis. Plant $J$ 55: $152-160$.
Ohyama K, Shinohara H, Ogawa-Ohnishi M, Matsubayashi Y. 2009. A glycopeptides regulating stem cell fate in Arabidopsis thaliana. Nat Chem Biol 5: 578-580.

Perales M, Reddy GV. 2012. Stem cell maintenance in shoot apical meristem. Curr Opin Plant Biol 15: 10-16.

Plotnikova JM, Rahme LG, Ausubel FM. 2000. Pathogenesis of the human opportunistic pathogen Pseudomonas aeruginosa PA14 in Arabidopsis. Plant Physiol 124: 1766-1774.

Qi Z, Verma R, Gehring C, Yamaguchi Y, Zhao Y, Ryan CA, Berkowitz GA. 2010. $\mathrm{Ca}^{2+}$ signaling by plant Arabidopsis thaliana Pep peptides depends on AtPepR1, a receptor with guanylyl cyclase activity, and cGMP-activated $\mathrm{Ca}^{2+}$ channels. Proc Natl Acad Sci 107: 21193-21198.

Shan L, He P, Li J, Heese A, Peck SC, Nürnberger T, Martin GB, Sheen J. 2008. Bacterial effectors target the common signaling partner BAK1 to disrupt multiple MAMP receptor-signaling complexes and impede plant immunity. Cell Host Microbe 4: 17-27.

Shapk ED, McAbee JM, Pillitteri LJ, Torii KU. 2005. Stomatal patterning and differentiation by synergistic interactions of receptor kinases. Science 309: 290-293.

Shiu S-H, Bleecker AB. 2003. Expansion of the receptor-like kinase/pelle gene family and receptor-like protein in Arabidopsis. Plant Physiol 132: 530-543.

Shiu SH, Karlowski WM, Pan R, Tzeng Y-H, Mayer KFX, Li W-H. 2004. Comparative analysis of the receptor-like kinase family in Arabidopsis and rice. Plant Cell 16: 1220-1234.

Song X-F, Yu D-L, Xu T-T, Ren S-C, Guo P, Liu C-M. 2012. Contributions of individual amino acid residues to the endogenous CLV3 function in shoot apical meristem maintenance in Arabidopsis. Mol Plant 5: 515-523.

Tena G, Boudsocq M, Sheen J. 2011. Protein kinase signaling networks in plant innate immunity. Curr Opin Plant Biol 14: $519-529$

Wang G, Fiers M. 2010. CLE peptide signaling during plant development. Protoplasma 240: 33-43.

Yamaguchi Y, Huffaker A, Bryan AC, Tax FE, Ryan CA. 2010. PEPR2 is a second receptor for the Pep1 and Pep2 peptides and contributes to defense responses in Arabidopsis. Plant Cell 22: 508-522.

Yamamizo C, Kuchimura K, Kobayashi A, Katou S, Kawakita K, Jones JDG, Doke N, Yoshioka H. 2006. Rewiring mitogen-activated protein kinase cascade by positive feedback confers potato blight resistance. Plant Physiol 140: 681692. 


\section{$\$_{\text {CSH\& }}^{\infty}$ Cold Spring Harbor Symposia SYMPOSIA On Quantitative Biology}

\section{Stem Cell Signaling in Immunity and Development}

H. Lee, O.-K. Chah, J. Plotnikov, et al.

Cold Spring Harb Symp Quant Biol 2012 77: 75-81 originally published online November 21, 2012

Access the most recent version at doi:10.1101/sqb.2012.77.014837

$\begin{array}{ll}\text { References } & \text { This article cites } 44 \text { articles, } 16 \text { of which can be accessed free at: } \\ \text { http://symposium.cshlp.org/content/77/75.full.html\#ref-list-1 }\end{array}$

License

Email Alerting Receive free email alerts when new articles cite this article - sign up in

Service the box at the top right corner of the article or click here. 